\title{
The contribution of diet preference to the disease course in children with familial Mediterranean fever: a cross-sectional study
}

\author{
Rabia Miray Kisla Ekinci ${ }^{1}$, Sibel Balci ${ }^{1}$, Atıl Bisgin ${ }^{2}$, Fatma Tugba Cetin ${ }^{3}$, Gokhan Tumgor ${ }^{4}$ \\ ${ }^{1}$ Department of Pediatric Rheumatology, Cukurova University Faculty of Medicine, Adana, Turkey \\ ${ }^{2}$ Cukurova University Adana Genetic Diseases Diagnosis and Treatment Center (AGENTEM) and Department of Medical Genetics, \\ Cukurova University Faculty of Medicine, Adana, Turkey \\ ${ }^{3}$ Department of Pediatrics, Cukurova University Faculty of Medicine, Adana, Turkey \\ ${ }^{4}$ Department of Pediatric Gastroenterology, Cukurova University Faculty of Medicine, Adana, Turkey
}

\begin{abstract}
Objectives: Familial Mediterranean fever (FMF) is characterized by recurrent, self-limiting attacks of fever and serositis. Nutrition is very important in the management of chronic diseases. Previous studies suggested that salty and fatty diet cause inflammation, therefore we aimed to investigate the effects of dietary self-efficacy and behavior about low-salt or low-fat diet on disease course in children with FMF.

Material and methods: This cross-sectional study included patients aged between 10-18 years, diagnosed in our department and admitted between June 2019 and September 2019. Demographic and clinical properties were obtained from the medical files of the patients. Children's Dietary Self-Efficacy Scale (CDSS) and Health Behavior Questionnaire (HBQ) - Diet Behavior Scale (DBS) were performed for dietary self-efficacy and behavior about preferring low-salt or low-fat diet. Clinical features were compared between patients, which were grouped according to the sum of these two scales, with a cut-off score of 5.

Results: The mean age of 74 FMF patients (44 females, 34 males), included in the study, was 14.6 \pm 2.82 years. Median CDSS and DBS scores of the patients were 5 (minimum -6 , maximum 14) and 0 (minimum -10, maximum 12), respectively. According to the sum of these two scales, 39 (52.7\%) patients who had scored at least 5 , had a statistically higher rate of complete response to colchicine. The remaining clinical parameters were similar between these two groups.

Conclusions: Low-salt or low-fat diet may be an adjuvant modification in the management of children with FMF. Further studies are needed to clarify the role of low-salt or low-fat diet in FMF pathogenesis.
\end{abstract}

Key words: colchicine, diet, familial Mediterranean fever, Mediterranean fever genotype, nutrition.

\section{Introduction}

Familial Mediterranean fever (FMF) is known as the most common hereditary autoinflammatory disease, which had been first genetically described [1]. Most of the patients had biallelic MEFV gene mutations, which is located in chromosome 16, and the disease-causing effects of these mutations were firstly defined in 1997.

In FMF patients, MEFV mutations are suggested to alter functions of the pyrin, commonly expressed in neutrophils, and thus lead to elevated amounts of interleukin 1 (IL-1) and inflammation [1, 2].

Address for correspondence:

Rabia Miray Kisla Ekinci, Department of Pediatric Rheumatology, Cukurova University Faculty of Medicine, 01330 Balcalı-Adana, Turkey, e-mail: mir_kisla@hotmail.com

Submitted: 17.02.2020; Accepted: 23.03.2020 
Patients with FMF, characterized by recurrent inflammation and serositis attacks lasting 1-3 days, usually present with fever, abdominal pain, arthralgia, chest pain and erysipelas-like erythema [3].

Colchicine is commonly prescribed to alleviate the frequency and severity of the symptoms and prevent the most common devastating complication, renal amyloidosis. Besides, some patients still require anti-IL-1 agents because of the colchicine resistance and intolerance $[4,5]$.

In recent years, the diet has been suggested to play an important role in the pathogenesis of multifactorial autoimmune diseases, including rheumatoid arthritis and systemic lupus erythematosus (SLE) [6-8]. Besides, we have recently showed an increased frequency of functional gastrointestinal disorders in FMF patients [9].

However, relevant literature lacks studies determining the effects of dietary habits on symptoms and treatment outcomes in FMF patients currently. Therefore, we aimed to investigate the dietary behavior and self-efficacy of children with FMF and the relation with symptoms, attack frequency, and treatment outcomes.

\section{Material and methods \\ Participants}

This retrospective cross-sectional study was performed in FMF patients, aged between 10-18 years and admitted to our department between June 2019 and September 2019. All patients were diagnosed according to Tel-Hashomer clinical criteria by the same pediatric rheumatologists and received colchicine treatment since their diagnosis [10].

Medical data including demographic properties, symptoms, laboratory results, MEFV gene analysis results, treatment and outcomes were obtained from the files of the patients retrospectively. Colchicine dosage, annual attack frequency and disease severity scores, established with Tel-Hashomer severity scoring system were also recorded [11].

Acute Phase Reactants (APRs), including erythrocyte sedimentation rate (ESR), C-reactive protein (CRP) and serum amyloid A (SAA) were obtained at the time of study enrollment. A complete response to colchicine was defined as having no attack during last year.

The study was approved by the Bioethical Committee at Cukurova University Faculty of Medicine.

\section{Questionnaires}

Dietary self-efficacy and behavior were assessed according to the Children's Dietary Self-Efficacy Scale (CDSS) and Health Behavior Questionnaire (HBQ) - Diet
Behavior Scale (DBS), covered by Child and Adolescent Trial for Cardiovascular Health (CATCH) trial [12].

Children's Dietary Self-Efficacy Scale questions the self-confidence about preferring less salty and fatty meals in their regular diets, rather than more salty and fatty meals. This scale consists of 15 items and was scored as -1 (not sure), 0 (a little sure) and 1 (very sure) for each item. Dietary behavior scale (DBS) consisted of 14 pictorial sub-items that make the participants choose a higher fat and higher sodium food or lower fat and lower sodium food. Each item was scored as -1 for unhealthy food and +1 for healthy food [13].

Both scales were self-administered in routine outpatient visit and the scores of CDSS and DBS were ranged between -15 and $+15,-14$ and +14 , respectively. Cut-off value for the sum of these two scores was considered as 5 . These scales were previously validated in Turkish children and relevant permission was obtained for this study from the corresponding investigators [13].

\section{Statistical analysis}

Categorical variables are measured as numbers and percentages, besides quantitative variables were analyzed as mean and standard deviation or median and minimum-maximum where appropriate.

Kolmogorov-Smirnov test was used for testing the distribution of the variables. Student $t$-test and Mann-Whitney U-tests were performed for comparison of quantitative variables, according to the distribution pattern. Spearman rho correlation coefficient was utilized while investigating the correlation between two continuous, abnormal distributed variables. Multivariate logistic regression analysis was utilized for determining the risk factors for a complete response to colchicine.

Statistical analysis was exerted by SPSS 20.0 statistical software package (IBM SPSS Statistics). The significance level for all tests was considered to be 0.05 .

\section{Results}

The mean age of the 74 patients ( 44 females, 34 males) was $14.6 \pm 2.82$ years at study enrollment. Median follow-up time was 37.8 (range, 12-133) months. Parental consanguinity and family history of FMF were present in 23 (31.1\%) and 32 (43.2\%) of the patients, respectively. The demographic and clinical features of the patients were summarized in Table I.

While investigating the distribution of MEFV mutations, we found that 18 (24.3\%) of the patients had M694V homozygosity (Table II). Median CDSS and DBS scores were 5 (minimum -6, maximum 14) vs. 0 (minimum -10, maximum 12), respectively. There was a low-degree positive correlation between CDSS and 
Table I. Main demographic and clinical features of the children with familial Mediterranean fever

\begin{tabular}{|lc|}
\hline Parameters & $n(\%)$ \\
\hline Age at symptom onset, year (mean \pm SD) & $5.01 \pm 3.41$ \\
\hline Age at diagnosis, year (mean \pm SD) & $9.04 \pm 3.38$ \\
\hline Age at study enrollment, year (mean \pm SD) & $14.58 \pm 2.82$ \\
\hline Gender, F/M & $44 / 30(59.5 / 40.5)$ \\
\hline Parental consanguinity & $23(31.1)$ \\
\hline Family history of FMF & $32(43.2)$ \\
\hline Recurrent fever & $67(90.5)$ \\
\hline Abdominal pain & $69(93.2)$ \\
\hline Arthralgia & $48(64.9)$ \\
\hline Arthritis & $15(20.3)$ \\
\hline Chest pain & $3(4.1)$ \\
\hline Myalgia & $2(2.7)$ \\
\hline Diarrhea & $6(8.1)$ \\
\hline Splenomegaly & $3(4.1)$ \\
\hline Erysipelas-like erythema & $6(8.1)$ \\
\hline Proteinuria & $2(2.7)$ \\
\hline Complete response to colchicine & $58(78.4)$ \\
\hline Total number of patients & $74(100)$ \\
\hline
\end{tabular}

FMF-familial Mediterranean fever, SD - standard deviation.

DBS scores $(r=0.374, p=0.001)$. CDSS and DBS were scored at least 0 (zero) in 59 (79.7\%) and 34 (45.9\%) of the patients.

When these two scores were summed-up, the patients were grouped according to a cut-off total score of 5. Regarding that, 39 (52.7\%) patients had self-efficacy and behaviors about healthy (less salty or less fatty) dietary meals. Demographic and clinical features were compared between these two groups in Table III. While demographic parameters, symptoms, attack frequency, colchicine dosage, disease severity and Acute Phase Reactants (APRS), including ESR, CRP and SAA were similar, complete response to colchicine was higher in FMF patients preferring less salty or less fatty meals.

Table IV indicated the results of multivariate logistic regression analysis of risk factors for complete response to colchicine, which showed the significance of the total dietary score on complete response to colchicine.

\section{Discussion}

In the present study, we found a statistically significant higher rate of complete colchicine response in patients with a preference of less salty or fatty meals. Besides, the symptoms and laboratory results did not differ between patients grouped according to their dietary
Table II. Distribution of MEFV genotype in children with familial Mediterranean fever

\begin{tabular}{|c|c|c|}
\hline MEFV genotype & Number $(n)$ & Percent (\%) \\
\hline M694V/M694V & 18 & 24.3 \\
\hline M694V/R202Q & 11 & 14.9 \\
\hline M694V/V726A & 4 & 5.4 \\
\hline M694V/E148Q & 2 & 2.7 \\
\hline M694V/R761H & 2 & 2.7 \\
\hline M694V/M680I & 2 & 2.7 \\
\hline M694V/K695R & 1 & 1.4 \\
\hline M694V/- & 2 & 2.7 \\
\hline M680I/M680I & 2 & 2.7 \\
\hline M680I/E148Q & 1 & 1.4 \\
\hline M680I/- & 4 & 5.4 \\
\hline M694I/M694I & 1 & 1.4 \\
\hline V726A/V726A & 1 & 1.4 \\
\hline V726A/R761H & 1 & 1.4 \\
\hline V726A/E148Q & 1 & 1.4 \\
\hline V726A/- & 1 & 1.4 \\
\hline E148Q/E148Q & 3 & 4.1 \\
\hline E148Q/R202Q & 2 & 2.7 \\
\hline E148Q/- & 5 & 5.4 \\
\hline Total & 74 & 100 \\
\hline
\end{tabular}

FMF-familial Mediterranean fever, MEFV - Mediterranean fever genotype.

self-efficacy and behavior. There are only a few ancient studies investigating the role of dietary fat consumption in FMF and none for dietary salt intake in FMF patients.

Firstly, in 1961, Mellinkoff et al. [14] suggested that low-fat diet greatly reduced the incidence of fever attacks in 8 patients with FMF. They also discovered that there was a close relationship between dietary indiscretions and prompt exacerbations. However, it was obvious why low-fat diet did improve the disease course in FMF patients in that study [14].

One year later, Sohar et al. [15] commenced a therapeutic trial on 32 colchicine-naïve FMF patients, of which 8 of them did not complete the regimen due to the belief of ineffectiveness. The authors concluded that a low-fat diet did not have an impact on the frequency of FMF attacks. Colchicine became the gold standard treatment option and no other studies have been carried for clarifying a beneficial effect of diet on FMF. Since then, the role of diet on FMF has been underestimated.

However, during recent years, diet and gut microbiota have been suggested to act as an important factor on pathogenesis and courses of several autoimmune dis- 
Table III. Comparison of main demographic and clinical features between children with familial Mediterranean fever according to their dietary self-efficacy and habits about preference of salty and fatty meals

\begin{tabular}{|c|c|c|c|}
\hline \multirow[t]{2}{*}{ Parameters } & \multicolumn{2}{|c|}{ Total score of dietary self-efficacy and behavior scales } & \multirow[t]{2}{*}{$p$} \\
\hline & $<5(n=35)$ & $\geq 5(n=39)$ & \\
\hline Age at symptom onset (year), mean $\pm \mathrm{SD}$ & $4.72 \pm 3.28$ & $5.26 \pm 3.55$ & 0.505 \\
\hline Age at diagnosis (year), mean $\pm \mathrm{SD}$ & $9.40 \pm 3.63$ & $8.72 \pm 3.16$ & 0.397 \\
\hline Age at study enrollment (year), mean \pm SD & $14.56 \pm 3.14$ & $14.59 \pm 2.25$ & 0.956 \\
\hline Gender, F/M, n (\%) & $19 / 16(54.3 / 45.7)$ & $25 / 14(64.1 / 35.9)$ & 0.479 \\
\hline Recurrent fever, $n(\%)$ & $33(94.3)$ & $34(87.2)$ & 0.435 \\
\hline Abdominal pain, $n(\%)$ & $34(97.1)$ & $35(89.7)$ & 0.361 \\
\hline Arthralgia, $n(\%)$ & $26(74.3)$ & $22(56.4)$ & 0.145 \\
\hline Arthritis, $n(\%)$ & $8(22.9)$ & $7(17.9)$ & 0.773 \\
\hline Chest pain, $n(\%)$ & $1(2.9)$ & $2(5.1)$ & 0.541 \\
\hline Diarrhea, $n(\%)$ & $3(8.6)$ & $3(7.7)$ & 0.609 \\
\hline Complete response to colchicine, $n$ (\%) & $23(65.7)$ & $35(89.7)$ & $0.022^{*}$ \\
\hline Colchicine dosage (mg/day), median (range) & $1(0.5-2)$ & $1(0.5-1.5)$ & 0.084 \\
\hline Disease severity score, median (range) & $6(4-10)$ & $6(4-11)$ & 0.920 \\
\hline Attack frequency per year, median (range) & $0(0-12)$ & $0(0-24)$ & 0.101 \\
\hline ESR (mm/h), median (range) & $14(2-47)$ & $18(2-68)$ & 0.881 \\
\hline CRP (mg/l), median (range) & $2.2(1-79.7)$ & $2.5(1-90.6)$ & 0.223 \\
\hline SAA (mg/l), median (range) & $4.1(2.9-763)$ & $5.3(2.8-349)$ & 0.698 \\
\hline
\end{tabular}

$C R P-C$-reactive protein, ESR - erythrocyte sedimentation rate, FMF-familial Mediterranean fever, SAA - serum amyloid A, SD - standard deviation, Student t-test and Mann-Whitney U-tests were utilized for comparison of demographic and laboratory data, respectively, * significant $p$-values $(<0.05)$.

eases. Dietary intake of vitamin A, D, E, polyunsaturated fatty acids and phytoestrogens have been proposed to have an appositive impact on SLE [7].

Robinson et al. [16] recently showed that the majority of SLE patients did not have talked to their health professionals about diet, however again the majority of them also believed that diet can be important in controlling disease symptoms [16].

On the other hand, although FMF pathogenesis had been enlightened with the presence of autosomal recessive inheritance pattern, patients even with low penetrance mutations in MEFV gene, heterozygote disease - causing mutations or no mutations have been seldom diagnosed with FMF [17].

By this point, without any evidence, diversity of genotype led us to think that there may be several environmental factors which effect the pathogenesis and the course of FMF. However, dietary factors have not been investigated in FMF patients to the best of our knowledge so far.

Recently, an experimental study suggested that higher salt intake results in the differentiation of Thelper (Th) cells to proinflammatory Th17 subtypes $[8,18]$. Another study also revealed that salt leads to epigenetic changes including DNA demethylation in Th cells and thus causes autoimmunity [19]. Higher salt concentration was also related to excessive secretion of proinflammatory cytokines from macrophages, overactivation in dendritic cells and overexpression of IL-1 3 [20].

Overall, these studies all proposed a low-grade inflammation theory in salty microenvironment. Therefore, although we could not demonstrate a bench-to-bedside clarification, we showed that complete colchicine response was statistically higher in patients with a higher self-efficacy and better behavior on preferring lower salty and fatty meals. We speculate that lower salt intake may alleviate the inflammatory state and lead to better disease courses in FMF patients.

On the other hand, it is an area of interest whether dietary fat and carbohydrate content have an impact on inflammation [21]. A relevant study did reveal higher plasma IL-6 and tumor necrosis factor-alpha (TNF- $\alpha$ ) levels after a meal rich in saturated fatty acids, and lower plasma levels of the same cytokines after a meal rich in polyunsaturated fatty acids [22]. Another study showed that fat-rich meals, regardless of the type of fatty acids, lead to elevated IL-6 but have no impact on TNF- $\alpha$ [23]. 
Table IV. Multivariate logistic regression analysis of risk factors for complete response to colchicine

\begin{tabular}{|lccccccc|}
\hline Risk factor & $\begin{array}{c}\text { Regression } \\
\text { coefficient }\end{array}$ & $\begin{array}{c}\text { Standard } \\
\text { error }\end{array}$ & Wald & $p$ & OR & \multicolumn{2}{c|}{ 95\% Cl of OR } \\
\hline Gender & -0.111 & 0.692 & 0.026 & 0.873 & 0.895 & 0.230 & 3.476 \\
\hline Age at symptom onset & 0.185 & 0.105 & 3.107 & 0.078 & 1.203 & 0.980 & 1.477 \\
\hline Age at diagnosis & -0.051 & 0.101 & 0.254 & 0.614 & 0.950 & 0.779 & 1.159 \\
\hline Fever & 0.729 & 1.385 & 0.277 & 0.599 & 2.073 & 0.137 & 3.126 \\
\hline Abdominal pain & 0.606 & 1.681 & 0.130 & 0.719 & 1.833 & 0.068 & 4.942 \\
\hline Arthralgia & 1.086 & 0.816 & 1.770 & 0.183 & 2.962 & 0.598 & 14.667 \\
\hline Arthritis & 0.272 & 1.021 & 0.071 & 0.790 & 1.312 & 0.177 & 9.713 \\
\hline M694V homozygosity & -0.379 & 0.906 & 0.175 & 0.676 & 0.684 & 0.116 & 4.043 \\
\hline Total score $\geq 5$ (CDSS + DBS) & 1.512 & 0.682 & 4.919 & $0.027^{*}$ & 0.220 & 0.058 & 0.839 \\
\hline Constant & -3.108 & 2.452 & 1.607 & 0.205 & & & \\
\hline
\end{tabular}

CDSS - Children's Dietary Self-Efficacy Scale, CI - confidence interval, DBS - Diet Behavior Scale, OR - odds ratio, * significant p-values.

These studies also suggested that a fatty diet may be linked to an inflammatory state similar to the results of our preliminary study. Although APRs did not differ between patients in terms of dietary behavior and self-efficacy, this result may be due to the small number of the patients and the lack of quantitative measurement of salt and fat in the meals.

There is much more need for more comprehensive studies on this topic for clarifying the impact of high-fat diet consuming on inflammation in FMF patients. Limitations of our study include a small number of participants and the lack of experimental bench-to-bedside study design. However, we think that our observational study will precede further researches on this topic.

\section{Conclusions}

In conclusion, dietary self-efficacy and behavior on a low-salt or fat intake are linked to higher rates of complete colchicine response. We think that dietary low-salt and fat may alleviate the low-grade inflammation and might be an important modification adjuvant to standard treatment in FMF patients. There is an ongoing need for further studies for clarifying the role of diet on the pathogenesis and course of FMF, which have been underestimated so far.

\section{Acknowledgments}

We sincerely thank to our recently passed mentor, Professor Mustafa Yilmaz, who shared his experience on Pediatric Rheumatology with us and encouraged us in academic writing. We also thank the patients and the families that have enrolled. We also recognize the con- tribution of our laboratory staff for their considerable efforts in providing comprehensive testing.

The authors declare no conflict of interest.

\section{References}

1. Ancient missense mutations in a new member of the RoRet gene family are likely to cause familial Mediterranean fever. The International FMF Consortium. Cell 1997; 90: 797-807, DOI: 10.1016/s0092-8674(00)80539-5.

2. Pathak S, McDermott MF, Savic S. Autoinflammatory diseases: update on classification diagnosis and management. J Clin Pathol 2017; 70: 1-8, DOI: 10.1136/jclinpath-2016-203810.

3. Kişla Ekinci RM, Balci S, Ufuk Altintaş D, Yilmaz M. The Influence of Concomitant Disorders on Disease Severity of Familial Mediterranean Fever in Children. Arch Rheumatol 2017; 33: 282-287, DOI: 10.5606/ArchRheumatol.2018.6488.

4. Ozen S, Demirkaya E, Erer B, et al. EULAR recommendations for the management of familial Mediterranean fever. Ann Rheum Dis 2016; 75: 644-651, DOI: 10.1136/annrheumdis-2015-208690

5. Kisla Ekinci RM, Balci S, Dogruel D, et al. Canakinumab in Children with Familial Mediterranean Fever: A Single-Center, Retrospective Analysis. Pediatr Drugs 2019: 21: 389-395, DOI: 10.1007/s40272-019-00354-6.

6. Rambod M, Nazarinia M, Raieskarimian F. The impact of dietary habits on the pathogenesis of rheumatoid arthritis: a case-control study. Clin Rheumatol 2018; 37: 2643-2648, DOI: 10.1007/s10067-018-4151-x.

7. Constantin MM, Nita IE, Olteanu R, et al. Significance and impact of dietary factors on systemic lupus erythematosus pathogenesis. Exp Ther Med 2019; 17: 1085-1090, DOI: 10.3892/etm.2018.6986.

8. Scrivo R, Perricone C, Altobelli A, et al. Dietary Habits Bursting into the Complex Pathogenesis of Autoimmune Diseases: The 
Emerging Role of Salt from Experimental and Clinical Studies. Nutrients 2019; 11: e1013, DOI: 10.3390/nu11051013.

9. Ekinci RMK, Balcı S, Akay E, et al. Frequency of functional gastrointestinal disorders in children with familial Mediterranean fever. Clin Rheumatol 2019; 38: 921-926, DOI: 10.1007/ s10067-019-04452-1.

10. Livneh A, Langevitz P, Zemer D, et al. Criteria for the diagnosis of familial Mediterranean fever. Arthritis Rheum 1997; 40 1879-1885, DOI: 10.1002/art.1780401023.

11. Sohar E, Gafni J, Pras M. Tel Hashomer key to severity score for FMF. In: Proceedings of the first international conference of FMF, eds. Sohar E, Gafni J, Pras M. Freund Publishing House, London, Tel Aviv 1997: 208.

12. Perry CL, Sellers DE, Johnson C, et al. The Child and Adolescent Trial for Cardiovascular Health (CATCH): Intervention, implementation, and feasibility for elementary schools in the United States. Health Educ Behav 1997; 24: 716-735.

13. Haney MO, Erdogan S. Factors related to dietary habits and body mass index among Turkish school children: a Cox's interaction model-based study. J Adv Nurs 2013; 69: 1346-1356, DOI: 10.1111/j.1365-2648.2012.06126.x.

14. Mellinkoff SM, Schwabe AD, Lawrence JS. A Dietary Treatment for Familial Mediterranean Fever. Arch Intern Med 1961; 108 80-85, DOI: 10.1001/archinte.1961.03620070082010.

15. Sohar E, Gafni J, Chaimow M, et al. Low-Fat Diet in Familial Mediterranean Fever. A Therapeutic Trial. Arch Intern Med 1962; 110: 150-154, DOI: 10.1001/archinte.1962.03620200010003.

16. Robinson GA, Mcdonnell T, Wincup C, et al. Diet and lupus: what do the patients think? Lupus 2019; 28: 755-763, DOI: 10.1177/0961203319845473.
17. Kisla Ekinci RM, Balci S, Dogruel D, et al. Twenty-Year Experience of a Single Referral Center on Pediatric Familial Mediterranean Fever: What Has Changed Over the Last Decade? J Clin Rheumatol 2019. DOI: 10.1097/RHU.0000000000001146 [Epub ahead of print].

18. Kleinewietfeld $M$, Manzel A, Titze J, et al. Sodium chloride drives autoimmune disease by the induction of pathogenic TH17 cells. Nature 2013; 496: 518-522, DOI: 10.1038/nature11868.

19. Wu H, Huang X, Qiu H, et al. High salt promotes autoimmunity by TET2-induced DNA demethylation and driving the differentiation of Tfh cells. Sci Rep 2016; 6: 28065, DOI: 10.1038/ srep28065.

20. Barbaro NR, Foss JD, Kryshtal DO, et al. Dendritic Cell Amiloride-Sensitive Channels Mediate Sodium-Induced Inflammation and Hypertension. Cell Rep 2017; 21: 1009-1020, DOI: 10.1016/j.celrep.2017.10.002.

21. Minihane AM, Vinoy S, Russell WR, et al. Low-grade inflammation, diet composition and health: current research evidence and its translation. Br J Nutr 2015; 114: 999-1012, DOI: 10.1017/S0007114515002093.

22. Masson CJ, Mensink RP. Exchanging saturated fatty acids for $(n-6)$ polyunsaturated fatty acids in a mixed meal may decrease postprandial lipemia and markers of inflammation and endothelial activity in overweight men. J Nutr 2011; 141: 816821, DOI: 10.3945/jn.110.136432.

23. Manning PJ, Sutherland WH, McGrath MM, et al. Postprandial cytokine concentrations and meal composition in obese and lean women. Obesity (Silver Spring) 2008; 16: 2046-2052, DOI: 10.1038/oby.2008.334. 